$\stackrel{N / N}{=}$

Global burnals Inc.

if

\title{
A Comparitive Study between Kapandji and Extra-Focal Fixation in Extra Articular Distal Radius Fractures
}

\author{
By Dr. Kautilyakumar V. Mahida, Dr. Jyotish G. Patel \& Dr. Ankit Patel \\ GCS Medical College Hospital \& Research Centre
}

Abstract- Purpose: To assess and compare the radiological and functional outcome of extra articular distal radius fractures treated by either Kapandji technique or extra-focal technique of $\mathrm{K}$ wire fixation.

Method: From January 2018 to March 2020, 60 patients with extra articular distal radius fracture were included in this prospective study after obtaining informed consent regarding the same. Out of these 30 went under extra-focal technique and 30 went under Kapandji technique of $\mathrm{K}$ wire fixation. After surgery in both groups we immobilized the limb in below elbow cast for 5 weeks after which cast was removed and k-wires were removed. Radiographs were taken at 1 month, 3 months and 6 months post operatively. All patients followed proper physiotherapy protocol after 5 weeks of surgery.

Keywords: extra articular distal radius fracture, extra-focal technique, kapandji technique, $K$ wire fixation, gartland and werley score.

GJMR-H Classification: NLMC Code: WE 175

Strictly as per the compliance and regulations of:

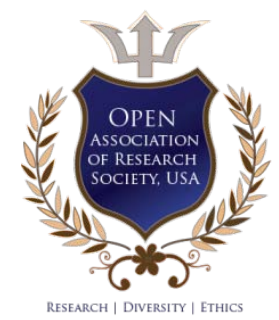

(C) 2021. Dr. Kautilyakumar V. Mahida, Dr. Jyotish G. Patel \& Dr. Ankit Patel. This research/review article is distributed under the terms of the Attribution-NonCommercial-NoDerivatives 4.0 International (CC BY-NC-ND 4.0). You must give appropriate credit to authors and reference this article if parts of the article are reproduced in any manner. Applicable licensing terms are at https://creativecommons.org/licenses/by-nc-nd/4.0/. 


\title{
A Comparitive Study between Kapandji and Extra-Focal Fixation in Extra Articular Distal Radius Fractures
}

\author{
Dr. Kautilyakumar V. Mahida ${ }^{\alpha}$, Dr. Jyotish G. Patel ${ }^{\sigma} \&$ Dr. Ankit Patel ${ }^{\rho}$
}

\begin{abstract}
Purpose: To assess and compare the radiological and functional outcome of extra articular distal radius fractures treated by either Kapandji technique or extra-focal technique of $\mathrm{K}$ wire fixation.

Method: From January 2018 to March 2020, 60 patients with extra articular distal radius fracture were included in this prospective study after obtaining informed consent regarding the same. Out of these 30 went under extra-focal technique and 30 went under Kapandji technique of $\mathrm{K}$ wire fixation. After surgery in both groups we immobilized the limb in below elbow cast for 5 weeks after which cast was removed and kwires were removed. Radiographs were taken at 1 month, 3 months and 6 months post operatively. All patients followed proper physiotherapy protocol after 5 weeks of surgery. Radiological parameters including Ulnar variance, Palmer tilt, Radial length and Radial inclination were measured in both groups in every patient in fractured and non fractured side and statistical comparison were made between the groups 6 months postoperatively. Functional outcomes were also assessed using Gartland and Werley score in both groups 6 months postoperatively.
\end{abstract}

Result: 6 months postoperatively Gartland and Werley score showed $43.33 \%$ excellent and $56.67 \%$ good results in kapandji technique; while it showed $40 \%$ excellent and $60 \%$ good result in extra-focal technique. Radiological parameters showed a comparable outcome in ulnar variance, radial length and radial inclination in both groups; while a statistically significant difference was seen in palmar tilt outcome of the two groups with Kapandji technique showing less loss of palmar tilt.

Conclusion: We conclude that both Kapandji and Extra-focal $\mathrm{K}$ wire fixation of Extra articular Distal Radius fracture provided excellent to good functional outcome as well as an acceptable radiological outcome at 6 months postoperative followup; given that appropriate immobilization followed by proper physiotherapy is followed by the patient.

Keywords: extra articular distal radius fracture, extra-focal technique, kapandji technique, $K$ wire fixation, gartland and werley score.

Author $\alpha$ : Senior Resident, Orthopaedics department, GCS Medical College Hospital \& Research Centre, Ahmedabad.

e-mail:kautilya18496@gmail.com

Author o: Head of the Department, Orthopaedics Department, GCS Medical College Hospital \& Research Centre, Ahmedabad.

e-mail:Drjgpatel@gmail.com

Author p: Assistant Professor, Orthopaedics department, GCS Medical College Hospital \& Research Centre, Ahmedabad.

e-mail:drankit_3103@yahoo.com

\section{INTRODUCTION}

( istal radius fracture is the most common fracture of the upper extremity ${ }^{(1)}$, accounting for $17.5 \%$ of all adult fractures. These are one of the most common skeletal injuries treated by orthopaedic surgeons and remains a topic of discussion till date, since Abraham Colles described this entity clinically in 1814.

Distal radius is important in kinematics of radiocarpal and radioulnar joints, proper reduction of articular surface, restoration of radial height, palmer tilt, radial inclination are prerequisites for good clinical outcome. Failure to achieve and maintain reduction leads to visible deformity, degenerative arthritis, distal radioulnar and radiocarpal instability and ulnar impaction syndrome with resultant pain, decrease in mobility, decrease grip strength and function. Distal radius is foundation of wrist joint and indispensable part of ligamentous support. So reconstruction of articular congruity and stable fixation reduce the incidence of post traumatic osteoarthritis and allows early functional rehabilitation.

Many treatment options for Distal radius fractures have been described, such as conservative treatment described by Colles ${ }^{(2)}$, close reduction and cast immobilization ${ }^{(3)}$, external fixation ${ }^{(4)}$, close reduction and percutaneous fixation with Kirschner wires ${ }^{(5)}$ and open reduction and internal fixation ${ }^{(6)}$.

There have been many studies regarding extra articular distal fracture treatment to find the better management option. Advantages of some methods over the other have become known; for example use of some kind of fixation like percutaneous fixation with Kirschner wires reduces the chances of the fracture from further displacement till the time of bone healing, which is a concern inclose reduction and cast immobilization ${ }^{(7)}$, but the gold standard method of treatment still is a matter of debate.

Close reduction and percutaneous fixation has the benefit of minor operation as compared to open reduction and internal fixation ${ }^{(7)}$ and it is much more economic for the patient compared to the plates that are used for open reductions. Percutaneous wire fixation has its own complications like pin site infections, pin breakage, tendon and nerve injury during wire insertion, 
still they are less numerous and less common compare to complications we may see in open reduction and internal fixation technique(8).

There are two distinct method of $\mathrm{K}$ wire fixation among the various methods which have been described which have gained popular acceptance. In one method $\mathrm{K}$ wires are inserted through the fracture sites and in other insertion is across the fracture sites. The former in which two or three wire are inserted through fracture sites and cross the opposite cortex, was first described by Kapandji in 1976 and various modification has been introduced since the ${ }^{(9)}$. The later also is done through many different modifications, of these one in which two $K$ wires are inserted through radius styloid and one through lunate articular facet has become more popular ${ }^{(10)}$.

Kapandji technique has some theoretical advantages as it provides a more dynamic fixation compared to Extrafocal technique which is supposedly more static $^{(11)}$. Although this may lead to a greater chance of collapse in Kapandji technique due to its dynamic nature but this has not been proven.

There has been little literature that compares the radiological and functional outcomes of these two technique, Hence the purpose of our prospective study is to analyze and compare the radiological and functional outcomes of Kapandji technique and Extrafocal technique of $\mathrm{K}$ wire fixation in Extra articular distal radius fractures.

\section{il. Materials and Methods}

This prospective study had been conducted in the Department of Orthopaedics, GCS Medical College and Research Hospital, Ahmedabad. From January 2018 to March 2020, 60 patients with extra articular distal radius fracture were included in this prospective study after obtaining informed consent regarding the same. Out of these 30 went under extra-focal technique and 30 went under Kapandji technique of $\mathrm{K}$ wire fixation. The exclusion criteria were comminuted fractures, open fractures, intra-articular extension, skeletally immature patient and fracture of duration more than 2 weeks. We randomly selected the method of fixation. Out of 30 patients in the Kapandji technique group, 14 (46.67\%) were male and 16 (53.33\%) were female and in the extra-focal fixation technique group 13 (43.33\%) were male and 17 (56.67\%) were female. Mean age in the Kapandji group was 49.33 years and in the extra-focal group it was 46.67 years.

\section{a) Technique}

The Kapandji technique was performed under general or local anaesthesia, the reduction was achieved by traction and counter traction method under fluoroscopic guidance. After making as tab wound within the first and second extensor compartment and mobilizing the under lying structures, first wire was inserted parallel and directly through the fracture site with caution not to damage the radial nerve and tendons. Then the wire was levered 45degree obliquely proximally and was drilled to the opposite cortex; this wire was occasionally also use to achieve radial height if traction alone provided in adequate height. Second and third wires inserted through third-forth and forth-fifth extensor compartment, respectively by the same method helping achieve and maintain the palmar tilt as well.

The extra-focal technique was performed under general or local anaesthesia, then close reduction was achieved by traction and counter traction and then with proper flexion the reduction was achieved in all cases under fluoroscopy guidance. After this, we make the first stab wound through first and second extensor compartment and after mobilization of underlying tendons and soft tissue we inserted the first $\mathrm{K}$ wire through radial styloid and cross the fracture site and go through the opposite cortex about 1-2 mm. The second $\mathrm{K}$ wire was passed from the lunate fossa cross the fracture site piercing the opposite cortex for about 1$2 \mathrm{~mm}$ and the third wire was again passed through the radial styloid cross the fracture site and go through the opposite cortex about 1-2 mm and distal to the piercing of the cortex done by the previous $\mathrm{k}$ wire inserted from the styloid. Preoperative, postoperative and 6 month follow up images of the 2 techniques is given in Figure 1.

After surgery in both groups we immobilized the limb in below elbow cast for 5 weeks after which cast was removed and k-wires were removed. Radiographs were taken at 1 month, 3 months and 6 months post operatively. All patients followed proper physiotherapy protocol after 5 weeks of surgery. Radiological parameters including Ulnar variance, Palmer tilt, Radial length and Radial inclination were measured in both groups in every patient in fractured and non fractured side and statistical comparison were made between the groups 6 months postoperatively. Functional outcomes were also assessed using Gartland and Werleyscore ${ }^{(12)}$ in both groups 6 months postoperatively. 
1)
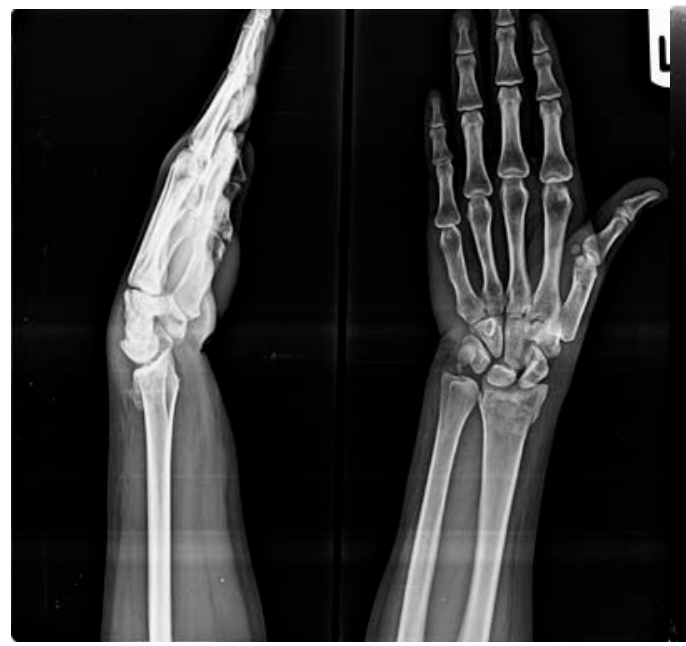

3)
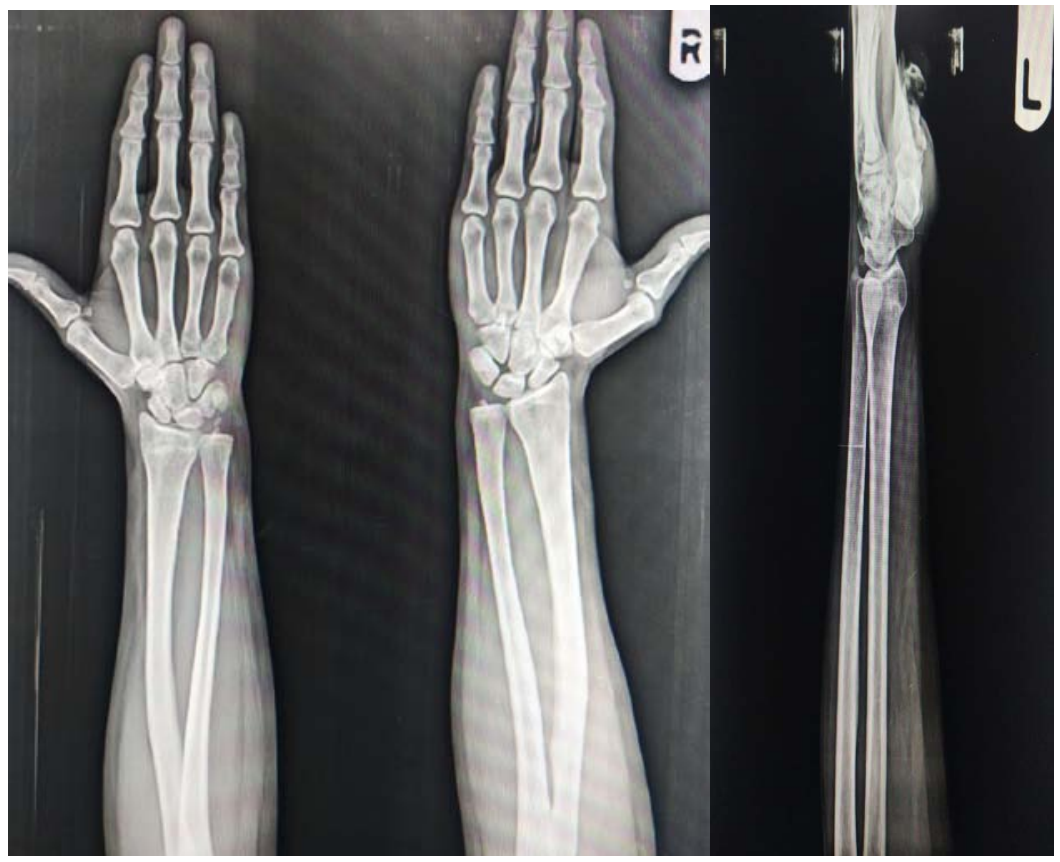

B.

1)
2)

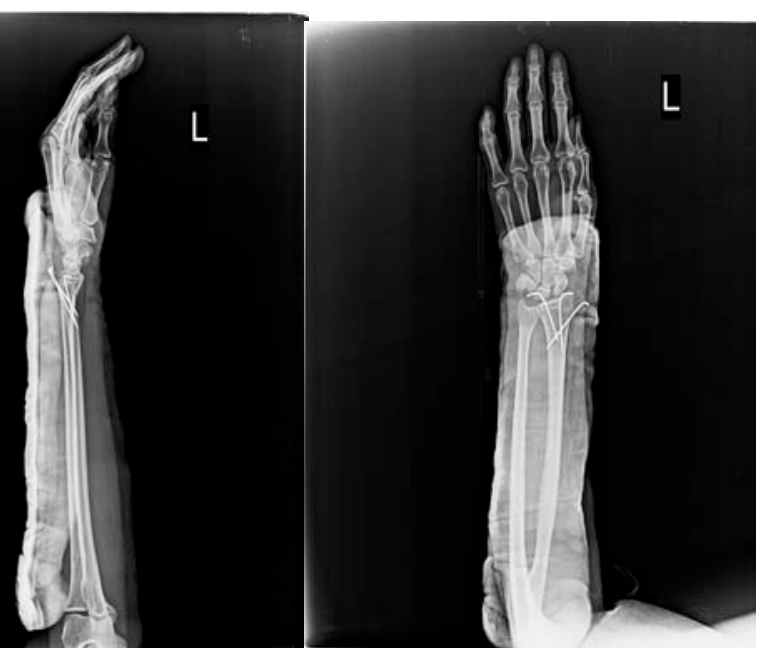

4)

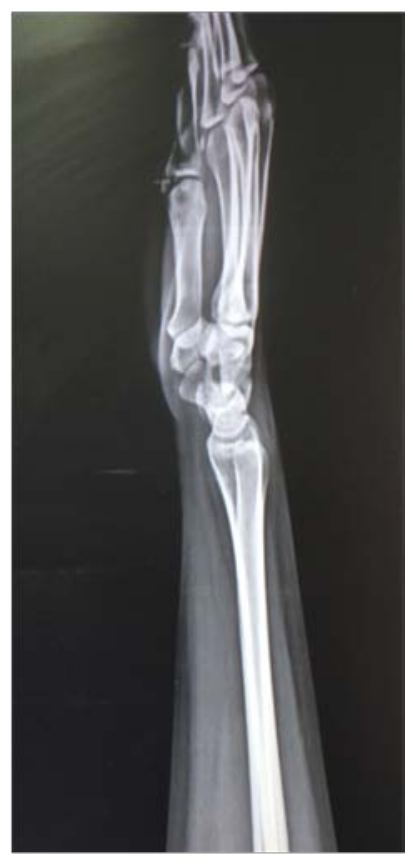

2)

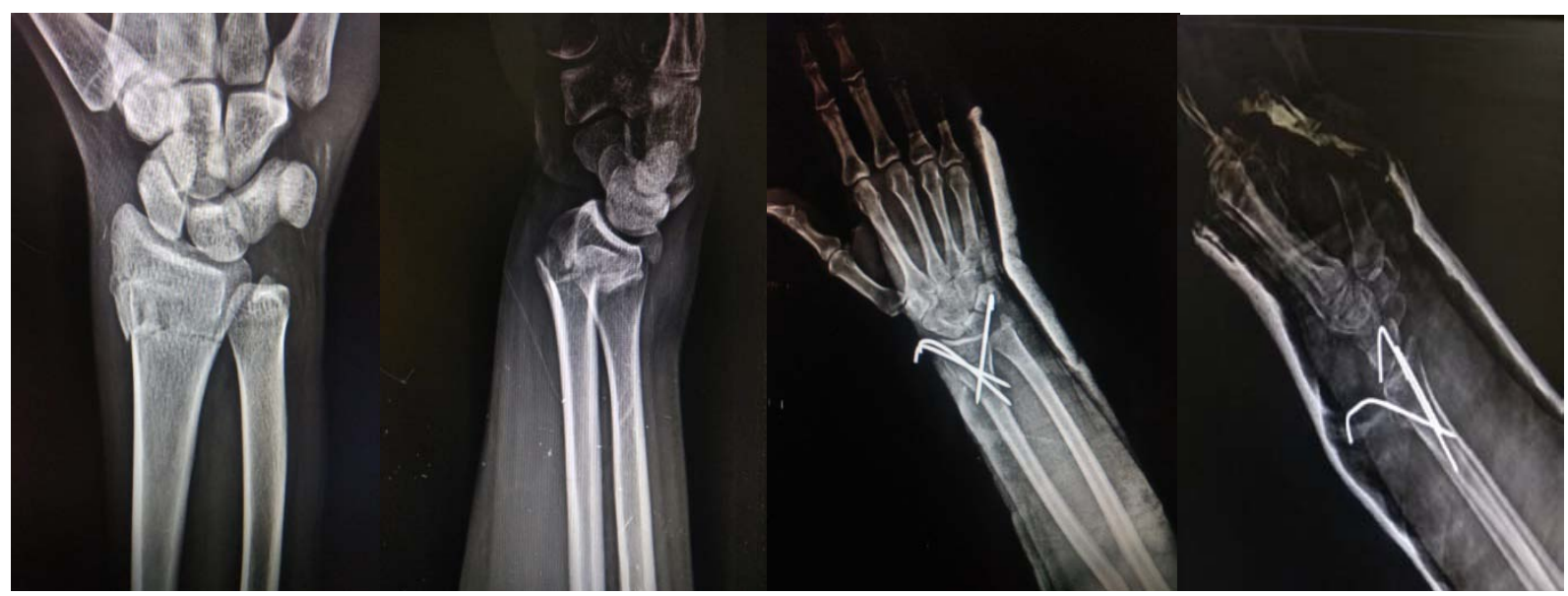


3)

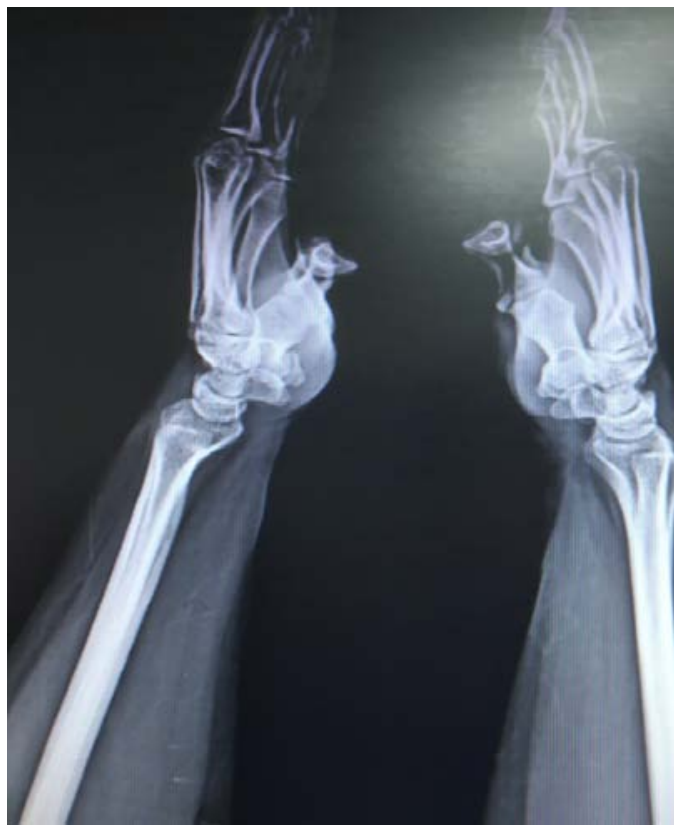

4)

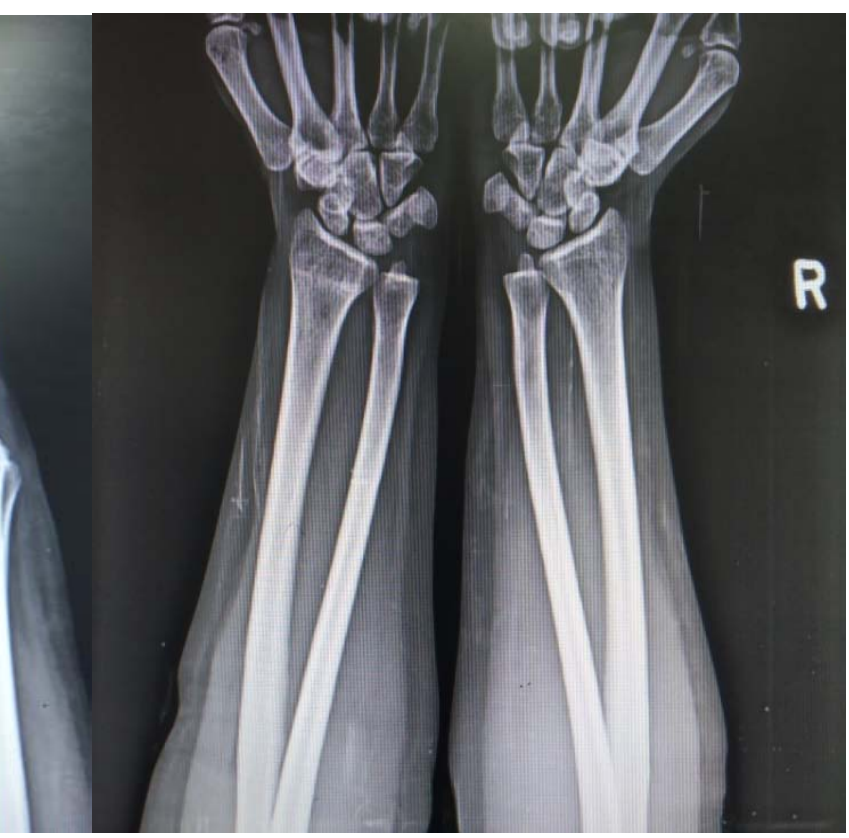

Figure 1: A. shows Kapandji technique 1) pre op radiographs, 2) immediate post op radiographs, 3), 4) 6 months follow up radiographs compared to opposite non fracture side. B. shows Extra Focal technique 1) pre op radiographs, 2) immediate post op radiographs, 3), 4) 6 months follow up radiographs compared to opposite non fracture side.

\section{Results}

From January 2018 to March 2020, 60 patients with extra articular distal radius fracture were included in this prospective study. Out of these, 30 were selected for Kapandji technique and other 30 for Extra focal technique. We had no complication during any surgery and there were no changes to plan after initiation of surgery. Close reduction was obtained in all cases and there were no wire breakage. No vascular, neurological or tendon related complications were noted post operatively.

Table 1: Summarizes the functional outcome of the two groups of extra articular distal radiuys fracture fixation using Gartland and Werly score at 6 months follow up post operatively.

\begin{tabular}{|c|c|c|}
\hline Gartland and Werley score & Kapandji Technique & Extra Focal Technique \\
\hline Excellent(0-2) & 13 & 12 \\
\hline Good(3-8) & 17 & 18 \\
\hline Fair(9-20) & 0 & 0 \\
\hline Poor(>20) & 0 & 0 \\
\hline
\end{tabular}

Radiological assessment was done in detail regarding 4 parameters namely Ulnar Variance, Palmar Tilt, Radial Length and Radial inclination. At 6 month follow up radiographs of every patient of the fractured and non fractured site was taken and the above mentioned parameters were measured. Mean and standard deviation for all parameters in both groups of study and of both fractured and non fractured limbs were calculated. The data was analyzed and compared. It was found that the distribution of data was non normal
Functional assessment was done at 6 months follow up in all cases. It was done using the Gartland and Werleyscore ${ }^{(12)}$. In the Kapandji group 13(43.33\%) patients showed excellent results while 17(56.67\%) patients showed good results at 6 months follow up while in Extra Focal group 12(40\%) patients showed excellent results while 18(60\%) patients showed good results. Table 1 summarizes the functional outcome. and hence appropriate statistic tests were used to compare the data.

The mean Ulnar variance in Kapandji group at 6 month follow up was $0.63 \mathrm{~mm}$ (standard deviation $0.54 \mathrm{~mm}$ ) on the fractured side and it was $0.67 \mathrm{~mm}$ (standard deviation $0.56 \mathrm{~mm}$ ) on the non fractured side while the mean Ulnar variance in Extra Focal group at 6 month follow up was $0.57 \mathrm{~mm}$ (standard deviation $0.54 \mathrm{~mm}$ ) on the fractured side and it was $0.67 \mathrm{~mm}$ (standard deviation $0.53 \mathrm{~mm}$ ) on the non fractured side. 
The mean Palmar tilt in Kapandji group at 6 month follow up was 11.2 degrees (standard deviation 0.66 degrees) on the fractured side and it was 11.33 degrees (standard deviation 0.61 degree) on the non fractured side while the mean Palmar tilt in Extra Focal group at 6 month follow up was 10.67 degree (standard deviation 0.96 degree) on the fractured side and it was 12 degree (standard deviation $0.59 \mathrm{~mm}$ ) on the non fractured side.

The mean Radial length in Kapandji group at 6 month follow up was $15 \mathrm{~mm}$ (standard deviation $1.78 \mathrm{~mm}$ ) on the fractured side and it was $16.33 \mathrm{~mm}$ (standard deviation $0.99 \mathrm{~mm}$ ) on the non fractured side while the mean Radial length in Extra Focal group at 6 month follow up was $14.33 \mathrm{~mm}$ (standard deviation $1.49 \mathrm{~mm}$ ) on the fractured side and it was $15.67 \mathrm{~mm}$ (standard deviation $0.96 \mathrm{~mm}$ ) on the non fractured side.

The mean Radial inclination in Kapandji group at 6 month follow up was 22.03 degrees (standard deviation 2.95 degrees) on the fractured side and it was 23.67 degrees (standard deviation 2.59 degrees) on the non fractured side while the mean Radial inclination in Extra Focal group at 6 month follow up was 22.93 degrees (standard deviation 2.08 degrees) on the fractured side and it was 24.67 degrees (standard deviation 1.61 degrees) on the non fractured side. Table 2 summarizes the various radiological findings.

Table 2: Summary of radiological findings at 6 month followup in both groups

\begin{tabular}{|c|c|c|c|c|c|}
\hline \multirow{2}{*}{$\begin{array}{c}\text { Radiological } \\
\text { Parameter }\end{array}$} & Limb side & \multicolumn{2}{|c|}{ Kapandji Technique } & \multicolumn{2}{c|}{ Extra Focal Technique } \\
\hline \multirow{3}{*}{$\begin{array}{c}\text { Ulnar Variance } \\
\text { (in mm) }\end{array}$} & Fractured & 0.63 & 0.54 & 0.57 & $\begin{array}{c}\text { Standard } \\
\text { deviation }\end{array}$ \\
\cline { 2 - 6 } & Non fractured & 0.67 & 0.56 & 0.67 & 0.54 \\
\hline \multirow{nyyyy}{*}{$\begin{array}{c}\text { Palmar Tilt } \\
\text { (in degrees) }\end{array}$} & Fractured & 11.2 & 0.66 & 10.67 & 0.53 \\
\cline { 2 - 6 } & Non fractured & 11.33 & 0.61 & 12.00 & 0.96 \\
\hline \multirow{2}{*}{$\begin{array}{c}\text { Radial Length } \\
\text { (in mm) }\end{array}$} & Fractured & 15.00 & 1.78 & 14.33 & 1.49 \\
\cline { 2 - 6 } & Non fractured & 16.33 & 0.99 & 15.67 & 0.96 \\
\hline $\begin{array}{c}\text { Radial Inclination } \\
\text { (in degrees) }\end{array}$ & Fractured & 22.03 & 2.95 & 22.93 & 2.08 \\
\cline { 2 - 6 } & Non fractured & 23.67 & 2.59 & 24.67 & 1.61 \\
\hline
\end{tabular}

These Radiological parameters in both methods were compared at 6 month follow up between the fractured and non fractured side by using Wilcoxon sign rank test to find any statistically significant difference.

It was found that there was a statistically significant difference between the findings of Radial length and Radial inclination between the fractured and non fractured side ( $p$ value $<0.05$ ) in Kapandji group suggesting a statistically significant loss of these parameters post surgery at 6 months follow up; though the difference in each patient's radiological parameter of fractured side compared to non fractured side remained acceptable(excellent to good range) as per the Sarmentio modification of Lindstorm criteria ${ }^{(13)}$.

It was found that there was a statistically significant difference between the findings of Palmar Tilt, Radial length and Radial inclination between the fractured and non fractured side ( $p$ value $<0.05$ ) in Extra Focal group suggesting a statistically significant loss of these parameters post surgery at 6 months follow up; though the difference in each patient's radiological parameter of fractured side compared to non fractured side remained acceptable(excellent to good range) as per the Sarmentio modification of Lindstorm criteria ${ }^{(13)}$.

The difference between the non fractured and the fractured side was calculated for every patient in each of the 4 above mentioned radiological parameters suggesting the loss for the said parameters at 6 months follow up; and the data so obtained in both groups was compared using Mann Whitney $U$ test. It was found that the loss of Ulnar variance, Radial length and Radial inclination in both groups was comparable but a statistically significant difference was found when comparing the loss of Palmar Tilt in both groups ( $p$ value $<0.05$ ); suggesting that in our study the loss of palmar tilt at 6 month follow up in Kapandji technique was significantly less than the loss of palmar tilt at 6 months follow up in Extra Focal technique.

\section{Discussion}

Distal end radius extra articular fracture is one of the commonest fractures that aorthopaedic surgeon has to treat. Although it is easy to diagnose there are many treatment options available like conservative cast management, $\mathrm{k}$ wire fixation by various methods, external fixator which may be joint spanning or non joint spanning, open reduction and internal fixation with plates and now recently augmentations in osteoporotic bones with light curable polymer and fixation has also been described. Given the wide selection among treatment options there still isn't any gold standard 
treatment option and no clear cut indications for selecting specific treatment options. Percutaneous $\mathrm{K}$ wire fixation remains the most commonly performed surgery for these fractures.

One of the first methods of wire fixation was done by Lambotte in 1908 that used 1 or 2 wire to fix the fracture through radial styloid ${ }^{(14)}$. Although the results were poor and revealed inability in maintaining the radial height but it did begin a long way of multiple methods of fixations that has lasted till now.

Cross wire fixation was introduced by Stein and Katz in 1975, who fixed the fracture with one wire through radius styloid and one through radius ulnar and dorsal cortex into volar cortex ${ }^{(15)}$. That method could maintain the radial inclination in $100 \%$, and radial length in $98.14 \%$ of the patients.

Kapandji described a technique in which he inserted wires inside the fracture side and drilled to the opposite cortex instead of conventional cross fixation wiring method ${ }^{(16)}$ but he did notrepor this results. Epinete in 1982 reported his series results with Kapandji technique in which there were 84\% excellent and good result ${ }^{(17)}$. Another study that assess the Kapandji technique was by Greating and Bishop in 1993 in Mayo Clinic in which they reported $84.6 \%$ excellent and good Mayo Clinic wrist score and also they advocated usage of this technique in fractures in which there is no volar cortex comminution or joint involvement. In our study it was found that Kapandji method gives 43.33\% excellent and $56.67 \%$ good results while Extra focal fixation method gives $40 \%$ excellent and $60 \%$ good results by Gartland and Werley score at 6 months follow up; Although the scores in both groups were comparable and the difference was not statistically significant.

Seyed Mehdi Mirhamidi et al in their prospective comparative study between kapandji and Extra focal $\mathrm{K}$ wire fixation reported that Palmar tilt were preserved better in the Kapandji group (12.41) than the extra-focal group (10.61) also it was not statistically significant but it may be because of the buttress effect of the second and third wire on the distal fragment ${ }^{(18)}$. In our study we found that the loss of palmar tilt on the fractured side opposed to non fractured side in the Kapandji group was less as compared to the loss in Extra Focal fixation and this difference was found to be statistically significant $(p$ value $<0.05)$

Its perceived that radial length is the most important radiologic and anatomic parameters that define the clinical outcome ${ }^{(19)}$ and it appears that any technique that maintain the radial length gives better functional result ${ }^{(15)}$. Our study showed comparable outcome in maintaining radial length and radial inclination in both Kapandji fixation and Extra focal fixation group and there was no statistically significant difference in these outcomes at 6 month follow up. Also in our study we found that in all the patient in both groups the radial length and radial inclination remained in excellent to good range as per the Sarmentio modification of Lindstormcriteria ${ }^{(13)}$.

In 1994 a comparative study between Kapandji technique and trans-radial wire technique, pain and Reflex Sympathetic Dystrophy (RSD) were more common in the Kapandji group but the range of motion was better in the Kapandji group till six weeks and after that became insignificant ${ }^{(20)}$. In our study 4 cases in Kapandji group developed RSD and 3 cases in Extra Focal group developed RSD. The range of motion in both groups were comparable at 6 months interval with no statistically significant difference.

One of the drawbacks of our study is that in order to have a common post operative protocol we immobilized the fractured limb in both the study groups for 5 weeks in below elbow cast so we were not able to replicate the early range of motion demonstrated by some studies in the Kapandji method of fixation ${ }^{(20)}$; although probably as a result of this we had fewer incidence of pain in follow up of patients in Kapandji group which became comparable to that of Extra Focal fixation group which is different from the result of other studies $^{(20)}$ showing higher incidence of pain in Kapandji Technique post operatively. Another limitation of our study is less number of patients and a lower follow up duration ( 6 months) which can be improved by a longer duration wider scale study.

\section{COnclusion}

We conclude that both Kapandji and Extra-focal $K$ wire fixation od Extra articular Distal Radius fracture provided excellent to good functional outcome as well as an acceptable radiological outcome at 6 months postoperative followup; given that appropriate immobilization followed by proper physiotherapy is followed by the patient.

\section{ACKnOWLedgements}

I would like to express my sincere gratitude to Dr. Jyotish G. Patel, Head of the department of Orthopaedics, Gcs Medical College, Hospital and Research Centre for his guidance, inspiration, teaching and valuable suggestion which helped in conducting this study.

I would like to acknowledge all the faculties and residents in the Orthopaedics department of our institute for their contribution in patient counselling, patient management, data collection and follow up.

I would like to thank Mr. Vasu G. Rathod (M.Sc. Statistics) for his expertise on the statistical analysis in this study.

I also would like to appreciate the patience and cooperation of all the patients and their families who took part in this study. I would also acknowledge Gcs Hospital and the Ethics committee for allowing me to conducting the study. 
Last but not the least I owe a lot to my parents, my wife, my sister and my family for their unconditional support, motivation, love, guidance in all the challenges of life striving to achieve my dreams.

\section{Compliance with Ethical Standards}

Conflict of interest: None declared

Ethical approval: The study was approved by the institutional ethics committee.

All patients gave an informed consent for participating in this study.

Funding: No funding sources

\section{References Références Referencias}

1. Fernandez DL, Martin CJ. Classification and conservative treatment of distal radius fractures. In: Watson HK, Weinzweig J, eds. The wrist. Philadelphia: Lippincott Williams \& Wilkins. 2001. pp: 277-298.

2. Colles A. Fractures of the carpal extremity of the radius. Edinb Med Surg J 1814; 10: 182-186.

3. Lidstrom A. Fractures of the distal end of theradius. A clinical and statistical study of end results. Acta Orthop Scand 1959; 30 suppl 41:1-118.

4. Cooney WP, Linscheid RL, Dobyns JH. External pin fixation for unstable Colles' fractures. J Bone Joint Surg Am 1979; 61: 840-845.

5. Brady O, Rice J, Nicholson P, Kelly E, O'Rourke SK. The unstable distal radial fracture one year post Kapandji intrafocal pinning. Injury 1999; 30: 251-255.

6. Orbay JL, Fernandez D. Volar fixation for dorsally displaced fractures of the distal radius: a preliminary report. J Hand Surg [Am] 2002; 27: 205-215.

7. Abbaszadegan $H$, Jonsson $U$, von Sivers $K$. Prediction of instability of Colles' fractures. Acta Orthop Scand 1989; 60: 646-650.

8. Kaempffe FA, Wheeler DR, Peimer CA, Hvisdak KS, Ceravolo J, Senall J. Severe fracture of the distal radius: effect amount and duration of external fixator distraction on outcome. J Hand Surg [Am] 1993; 18: 33-41.

9. Kapandji A. L'osterosynthese par double embrocharge intra-focal: traitement fonctionnel des fractures non articulares de l'extremite inferieure du radius. Ann Chir 1976; 30: 903- 908.

10. Rayhack JM. The history and evolution of percutaneous pinning of displaced distal radius fractures. OrthopClin North Am 1993; 24: 287-300.

11. Epinette J, Lehut J, Decoulx J. Pouteau-Colles' fractures: double intrafocal pinning according to Kapandji. Ann Chir Main 1982; 1: 71-83.

12. Changulani M, Okonkwo U, Keswani T, Kalairajah Y. Outcome evaluation measures for wrist and hand: which one to choose? Int Orthop. 2008 Feb; 32(1): 1-6. doi: 10.1007/s00264-007-0368-z. Epub 2007 May 30.
13. Sarmiento A, Pratt GAW, Berry NC, Sinclair WF. Colles' fractures: functional bracing in supination. J Bone Jt Surg. 1975; 57A: 311-317.

14. Fractures and Injuries of the Distal Radius and Carpus E-Book: The Cutting Edge-Expert Consult: Online and Print David J Slutsky, A Lee Osterman Elsevier Health Sciences, 2008.

15. Stein A, Katz S. Stabilization of comminuted fractures of the distal inch of the radius: Percutaneous pinning. ClinOrthop 1975; 108: 174- 181.

16. Kapandji A. L'osteosyntese par double embrochage intrafocal: Traitement fonctionnel des fractures non articulaires de l' extremite inferieure du radius. Ann Chir 1976; 30: 903

17. Epinete J, Lehut J, Decoulx J, et al. Fracture de Pouteau-Colles: double embrochage intrafocal en berceau selon Kapandji. A propos d'une se'rie homoge ' ne de soixante-douzecas. Ann Chir Main 1982; 1: 71.

18. Mirhamidi SM, Bayat FM. A prospective comparison between Kapandji and percutaneous extra-focal fixation in extra articular distal radius fractures. Int $J$ Clin Exp Med. 2013; 6(2): 133-139.

19. Trumble TE, Wagner W, Hanel DP, Vedder NB, Gilbert M. Intrafocal (Kapandji) pinning of distal radius fractures with and without external fixation. J Hand Surg [Am] 1998; 23: 381-394.

20. Lenoble E, Dumontier C, Goutallier D, Apoil A. A prospective comparison between trans styloid and kapandji fixations. J Bone Joint Surg (Br) 1995; 77B: 562-7. 